\title{
Hybrid three-stage repair of mega aorta with Lupiae technique: tips and tricks
}

\author{
Giampiero Esposito ${ }^{1}$, Antonio Pignatelli ${ }^{1}$, Gaetano Contegiacomo ${ }^{1}$, Giangiuseppe Cappabianca ${ }^{2}$, \\ Cesare Beghi ${ }^{2}$, Manuela Conte ${ }^{1}$, Micaela De Palo ${ }^{1}$, Carlo Lafranceschina ${ }^{1}$, Fabio Tiecco ${ }^{1}$, Francesco \\ Bartolomucci $^{3}$, Matteo Miccoli ${ }^{1}$, Massimiliano Conte ${ }^{1}$ \\ ${ }^{1}$ Department of Cardiovascular Diseases, Mater Dei Hospital, Bari, Italy; ${ }^{2}$ Department of Cardiac Surgery, Circolo Hospital, University of Insubria, \\ Varese, Italy; ${ }^{3}$ Department of Cardiology, “L. Bonomo” Hospital, Andria (BT), Italy \\ Correspondence to: Giampiero Esposito. Director of Department of Cardiovascular Diseases, Mater Dei Hospital, Bari, Italy. \\ Email: espositogp@hotmail.com.
}

\begin{abstract}
Mega aorta syndrome (MAS) poses a complex clinical challenge: the involvement of the ascending aorta, aortic arch, descending thoracic and abdominal aorta with extension below the origin of renal arteries requires almost total replacement of the aorta. The modality of treatment remains still controversial. Different aortic debranching techniques have been developed to re-route the origin of epiaortic and visceral vessels and achieve an optimal landing zone for implantation of subsequent endovascular grafts. We illustrate the Lupiae technique as a further evolution of the aortic debranching and hybrid repair of a mega aorta. It was developed with the purpose to exclude a very long segment of diseased aorta by implanting two or more endoprostheses between two surgically-generated landing zones. We describe a series of 27 patients treated by this hybrid three-stage mega-aorta repair; the tips and tricks discussed here facilitate a safe and effective procedure, enable treatment of frail patients and help to avoid life-threatening complications.
\end{abstract}

Keywords: Mega aorta; hybrid; debranching

Submitted Jan 25, 2018. Accepted for publication Apr 24, 2018.

doi: $10.21037 /$ acs.2018.05.04

View this article at: http://dx.doi.org/10.21037/acs.2018.05.04

\section{Introduction}

Mega aorta syndrome (MAS), originally described in 1960 by Cullhed et al. (1), is a rare entity that requires extensive repair procedures. Although open surgical repair of MAS has evolved greatly over the last decade, these procedures still demonstrate significant morbidity and mortality (2).

A hybrid approach to MAS, which integrates surgical debranching of epiaortic and visceral vessels with catheterbased repair technologies, has been developed to minimize surgical trauma, and enables complete exclusion of the diseased thoracoabdominal aorta (3). This is known as "Lupiae technique", named from the Latin name of the city (Lecce) where the procedure was developed, was initially attempted in 2006 and described in 2008 (4).

According to the MAS classification proposed by Esposito et al. in 2011 (5), MAS type III, where aneurysmal dilatation involves the entire aorta from the aortic valve to the iliac bifurcation (Figure 1) requires a three-stage repair. Stage I involves surgical replacement of the ascending aorta and proximal re-routing of the aortic arch vessels. The surgical replacement of the infrarenal aorta with distal re-routing of the visceral and renal vessels is performed in stage II, allowing subsequent complete exclusion of the residual diseased aorta between the surgically shaped proximal and distal Dacron landing zone (DLZ) in the endovascular stage III (6).

\section{Operative technique}

\section{Stage I}

\section{Preparation}

The patient is placed in a supine position and both left and 


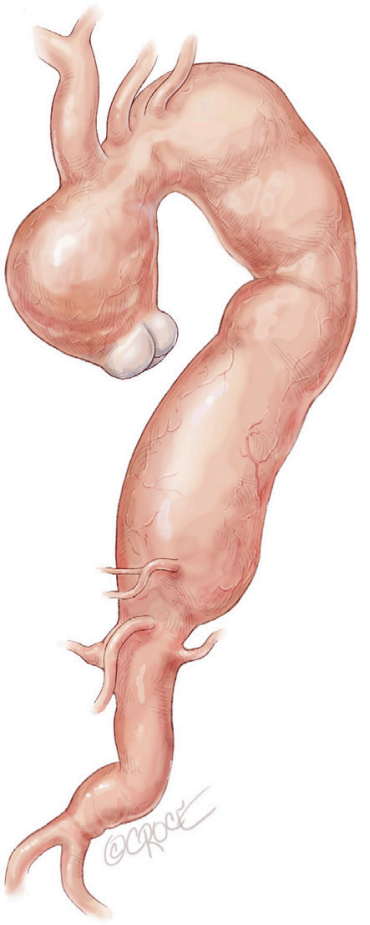

Figure 1 In MAS, type III the aneurismal dilatation involves the aorta entirely from the aortic valve to the iliac bifurcation. A three-stage repair is proposed for these patients. MAS, mega aorta syndrome.

right radial arteries are cannulated for continuous blood pressure monitoring. Transesophageal echocardiography and near infrared spectroscopy monitoring of cerebral oxygenation (INVOS Somanetics, Troy, MI, USA) are routinely used.

\section{Exposition}

Full or partial sternotomy at the 4th intercostal space is performed with the adjunct of a short left latero-cervical incision for better exposure of the origin of the neck vessels (Figure 2).

\section{Operation}

After systemic heparinization, the Innominate Trunk (IT) is side-clamped and an $8 \mathrm{~mm}$ Dacron graft is anastomosed in end-to-side fashion and connected to the main arterial line for central arterial perfusion. The site of this anastomosis on the IT should be very close to its origin from the arch to permit subsequent easier detachment and reimplantation of the IT in an arterial zone not used for this cannulation manoeuvre.

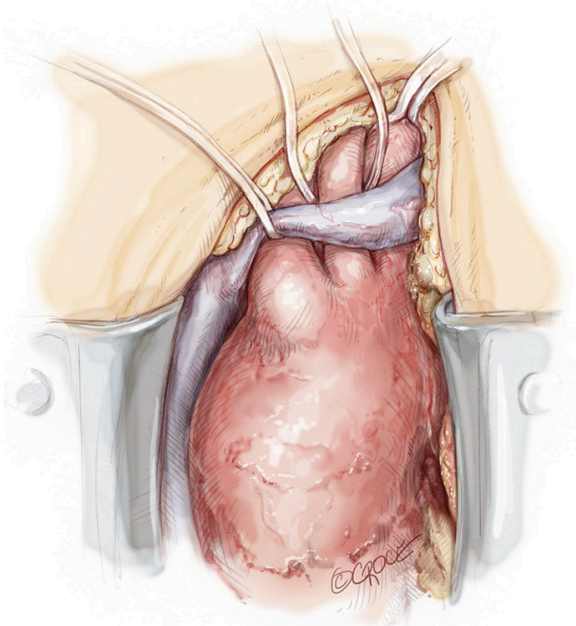

Figure 2 The proximal aorta is exposed through either a complete or a partial sternotomy at 4th intercostal space; in both case the wound is extended with a $2-3 \mathrm{~cm}$ left latero-cervical incision in order to improve the exposure of the neck vessels.

After atrio-caval cannulation by means of a two-stage venous cannula, the left ventricle is vented through the right superior pulmonary vein. Before instituting cardiopulmonary bypass (CPB), the left subclavian artery (LSA) is completely mobilized behind the left side of the left common carotid artery (LCCA), then divided at its origin from the aortic arch and anastomosed in end-to-end manner to a $10 \mathrm{~mm}$ Dacron graft using a 5-0 polypropylene suture. The Dacron graft is then connected to a selective arterial line perfusion $(7-10 \mathrm{~mL} / \mathrm{kg} / \mathrm{min})$ in order to improve cerebral and spinal cord perfusion throughout the procedure. The residual LSA stump on the arch is then oversewn with 3/0 polypropylene suture. Exposure, ligation and anastomosis of LSA is dramatically improved by a small left latero-cervical extension of the sternotomy (Figure 3). $\mathrm{CPB}$ is then initiated with main arterial line perfusion from the IT and selective perfusion of LSA.

The patient is cooled to achieve moderate hypothermia at $28-30^{\circ} \mathrm{C}$. The ascending aorta is cross-clamped proximal to the IT and either antegrade or a selective intracoronary cardioplegic infusion are administrated as appropriate. The ascending aorta is then resected, the aortic valve and the aortic root are inspected and repaired or replaced as deemed necessary. Once this step is performed, the multi-branched Dacron graft (GelweaveTM Lupiae Graft-Vascutek Ltd Scotland UK) is anastomosed to the native sinotubular junction using 4-0 polypropylene as preferred suture, 


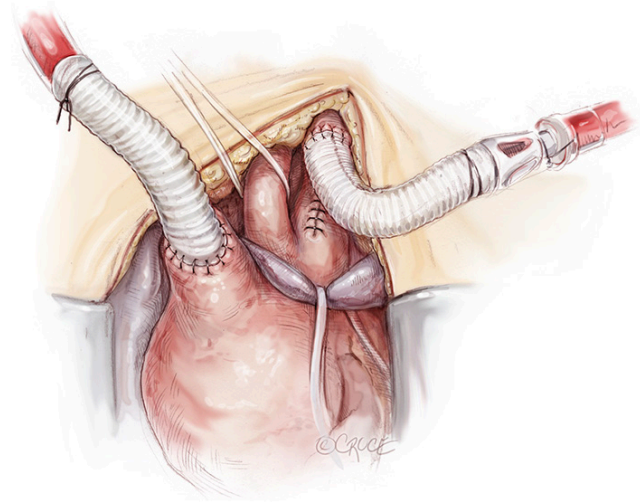

Figure 3 The main arterial cannulation site on the Innominate Trunk (IT), performed by direct cannulation or by $8 \mathrm{~mm}$ Dacron graft interposition, should be very close to its origin from the arch to permit a subsequent easier detachment and reimplantation of IT in an arterial zone not used for this cannulation manoeuvre. The residual LSA stump on the arch is then oversewn with $3 / 0$ polypropylene suture. Exposure, ligation and anastomosis of LSA is dramatically improved by the small left latero-cervical extension of the sternotomy. CPB is then initiated with main arterial line perfusion from the IT and selective perfusion of LSA. LSA, left subclavian artery; CPB, cardio-pulmonary bypass.

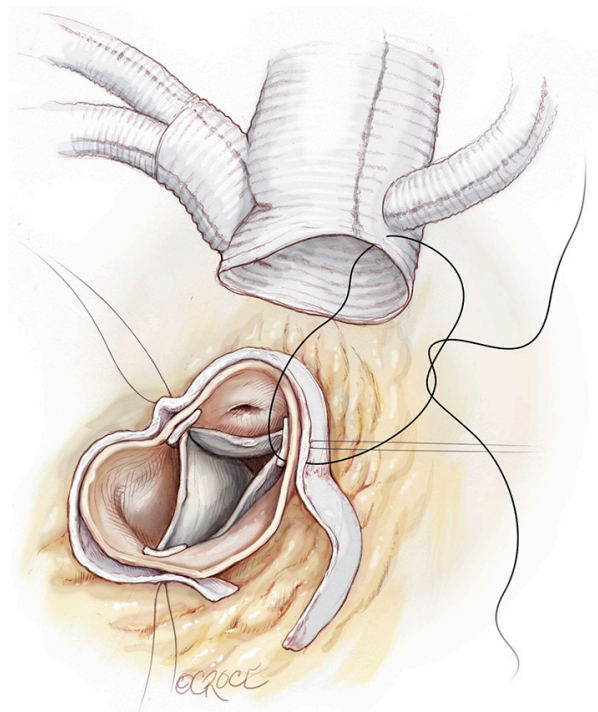

Figure 4 After ascending aorta cross-clamp and cardioplegia infusion, the diseased ascending aorta is removed and the multibranched Dacron graft (GelweaveTM Lupiae Graft, Vascutek Ltd., Scotland, UK) is anastomosed to the native sinotubular junction using a 4/0 polypropylene as preferred suture, leaving at least $1.0 \mathrm{~cm}$ of Dacron between the sinotubular junction and the emergency of the Lupiae graft side branches.

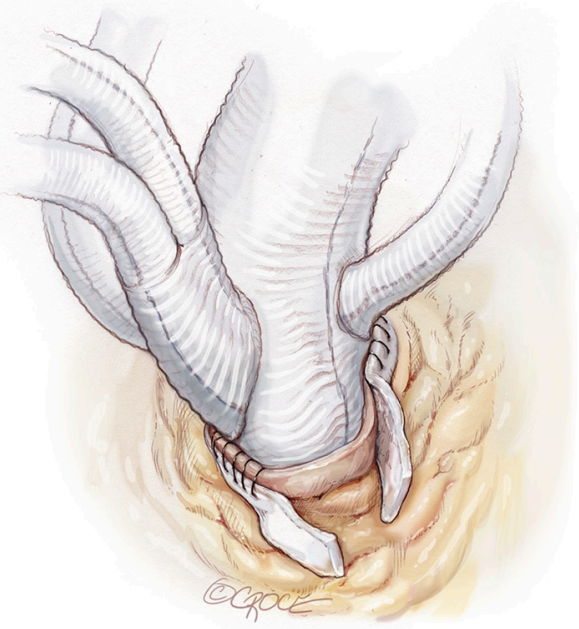

Figure 5 The side branches originating from the Lupiae graft take off from the main Dacron graft with a narrow $\left(30^{\circ}\right)$ angle; this allow them to point up toward the native neck vessels once the main prosthesis has been correctly implanted. A critical aspect is to implant the main Dacron graft positioning the "Lupiae" bovine trunk in the space between the aorta and SVC, in order to avoid neck vessels branches compression when the chest will be closed.

leaving at least $1.0 \mathrm{~cm}$ of Dacron between the sinotubular junction and the emergence of the Lupiae side branches (Figure 4).

It is very important that the orientation of the multibranched Dacron graft and its side branches are sutured in such a way to lie between the ascending aorta and the superior vena cava in order to avoid any kinking or compression of the rerouted epiaortic vessels after sternal closure. This is the reason why the side branches of the Lupiae graft take off from the main Dacron graft at a 30 -degree angle rather than the more common $90^{\circ}$ angle of other multi-branched grafts, having to be directed upward from the branches of the sinotubular junction toward the neck vessels (Figure 5). Once the proximal aortic anastomosis is completed, the distal aortic anastomosis is performed in Criado zone, maintaining the aortic crossclamp, or in Criado zone 1-2, declamping the aorta and performing a distal circulatory arrest according to the extension and nature of the aneurysmal pathology. The distal aortic anastomosis is performed using 4-0 polypropylene with or without Teflon felt reinforcement depending on the quality of the aortic wall. When circulatory arrest is needed, central arterial perfusion is transformed to selective antegrade cerebral perfusion using 


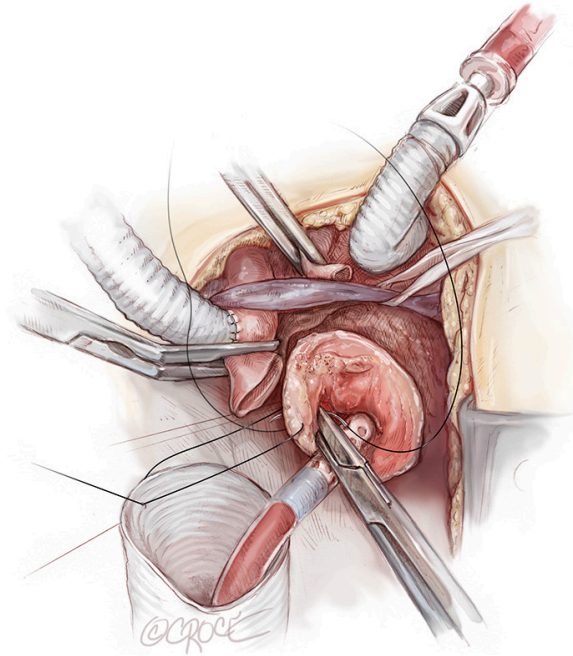

Figure 6 Once the proximal aortic anastomosis is completed, the distal aortic anastomosis can be performed either in Criado zone zero (maintaining the aortic cross-clamp in) or alternatively in Criado zone 1-2 (in open anastomosis, antegrade cerebral perfusion and distal circulatory arrest), according to the extension and nature of the aneurismal pathology. The distal aortic anastomosis is generally accomplished using 4/0 polypropylene suture with or without Teflon felt reinforcement according the quality of the aortic wall

the same arterial line connected to the IT, reducing the arterial flow to $10 \mathrm{~mL} / \mathrm{kg} / \mathrm{min}$ and clamping the IT origin (Figure 6).

The choice between unilateral and bilateral cerebral perfusion is based on pre-operative cerebral angiographic imaging of the circle of Willis, intra-operative INVOS data (as well as transcranial Doppler data when available) and on inspection of LCCA back-flow; if there is any doubt about the completeness of cerebral perfusion, bilateral cerebral perfusion is instituted using an adjunctive arterial line derived from the LSA-selective perfusion system, which is inserted in the LCCA.

When the distal aortic anastomosis is completed, systemic perfusion, which was previously located exclusively in the IT, is recommenced by connecting a bifurcation of the main arterial line to the $10 \mathrm{~mm}$ side-branch of the Lupiae graft originating from the inferior margin of the prosthesis.

Fine tuning of cerebral vascular resistance by strict control of $\mathrm{paCO}_{2}$ during $\mathrm{CPB}$, in the setting of multiple lines and blood flow modulation, is of paramount importance to optimize the quality of the antegrade cerebral perfusion. During this phase the unilateral or bilateral cerebral perfusion is continued and the re-warming phase is commenced.

Throughout all these surgical steps, the importance of clear and timely communication between surgeon, anesthesiologist and perfusionist should be emphasised. To complete the operation, the epi-aortic vessels are rerouted starting with the LCCA reimplantation, by an end-toend anastomosis with the $8 \mathrm{~mm}$ branch of the so-called Bovine-like-trunk of the Lupiae graft. The IT re-routing requires arrest of its selective perfusion by clamping the derived $\mathrm{Y}$ arterial line side-connected with the IT through the $10 \mathrm{~mm}$ interposition Dacron graft (previously used as a central arterial line and then as cerebral perfusion line). As previously explained, the proximal part of the IT involved in the arterial cannulation is resected and the IT is clamped just before its bifurcation into the right subclavian artery and right common carotid artery. The IT, prepared in this fashion, is easily anastomosed to the $10 \mathrm{~mm}$ branch of the Bovine-like-Trunk using a 5-0 polypropylene suture (Figure 7). At the end of cardiopulmonary bypass, the last epiaortic vessel to be re-implanted is the LSA, which had previously been detached, anastomosed to a $10 \mathrm{~mm}$ Dacron graft and perfused during the procedure (Figure 8). The LSA re-routing can be accomplished in two different ways: either by the third branch of the Bovine-like-trunk of the Lupiae graft or by its $10 \mathrm{~mm}$ left-sided lateral branch, previously used as the main arterial line. The choice between these different solutions depends on the pericardial space available and on the position of the LSA in comparison to the other re-routed epiaortic vessels.

\section{Completion}

At the end of the procedure, a well-performed stage I Lupiae technique is characterized by:

(I) Correct position of the origin of the re-routed epiaortic vessels from the Lupiae graft in order to avoid kinking or compression after sternal closure;

(II) DLZ of adequate length $(>3.5 \mathrm{~cm})$;

(III) Presence of radiopaque marker at the origin of the rerouted epiaortic vessels.

These features of the multi-branched Dacron graft implantation in the ascending aorta with debranching and re-routing of epiaortic vessels are critical to avoid any kinking or compression of the graft and its branches at sternal closure and to allow a safe, effective and easy deployment of the endovascular stent graft in the newly forged ascending aorta in the third endovascular stage. In 


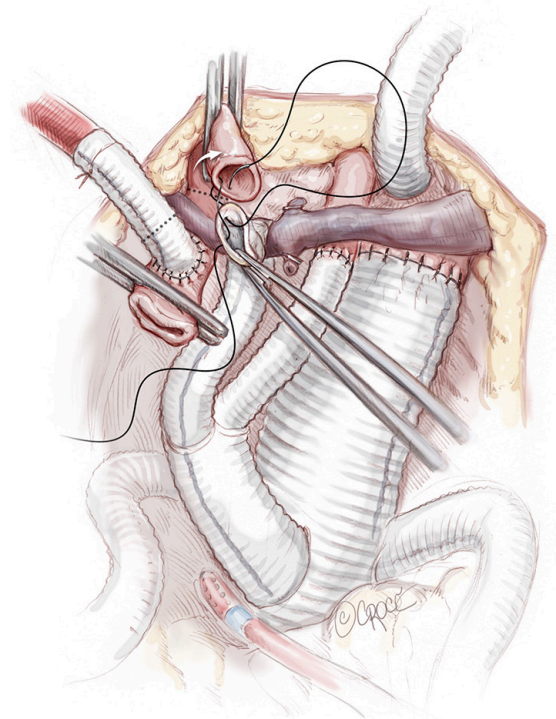

Figure 7 Rerouting of epiaortic vessels is performed: firstly, the LCCA is anastomosed end-to-end with the $8 \mathrm{~mm}$ division branch of the bovine trunk of the Lupiae graft. Once the cerebral perfusion is resumed via the rerouted LCCA, the perfusion line on the IT is disconnected and this vessel is resected more distally and anastomosed end-to-end to the $10 \mathrm{~mm}$ second division branch of the Lupiae bovine trunk. LCCA, left common carotid artery; IT, Innominate Trunk.

particular, it avoids complications such as the 'bird beaking' effect with consequent type 1 endoleak, if the landing zone is $<2 \mathrm{~cm}$, or partial obstruction of the branches, suggested by the absence of radiopaque markers (7-9).

\section{Stage II}

\section{Preparation}

Between 30 to 60 days after stage I, the patient is prepared as for routine abdominal vascular surgery with adjuncts of:

(I) Sigmoid tonometry to monitor mucosal ischemia and to guide intraoperative fluid infusions as large third space losses should be expected and prevented;

(II) Use of thermal pads to mitigate changes in body temperature changes.

It should be noted that the use of cerebrospinal fluid drainage and monitoring of somatosensory evoked potentials is not deemed necessary in any of MAS III repair.

\section{Operation}

A midline laparotomy is performed to expose the aorta

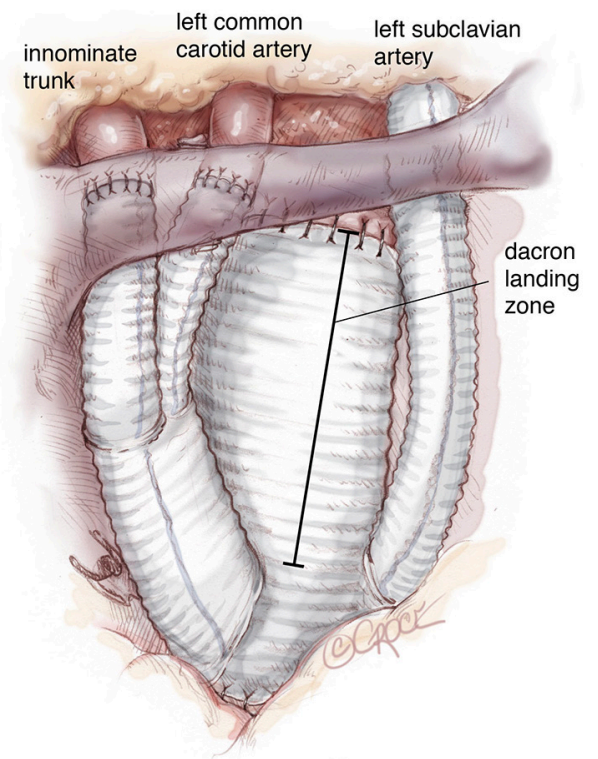

Figure 8 Once off by-pass, the LSA is re-routed by an end-to-end anastomosis with the side branch of the Lupiae graft. The adequate length of Dacron landing zone is highlighted. LSA, left subclavian artery.

together with its branches to be rerouted, including the visceral arteries, renal arteries and both common iliac arteries (Figure 9). Firstly, the celiac trunk (CT) is prepared in the retroepiploic cavity after mobilization and twisting of the left hepatic lobe to the right by transection of the left triangular ligament. Isolation of the CT should go so far as to allow ligation at its origin from the aorta. During this phase, one must be cautious of the pre-aortic lymph nodes surrounding the $\mathrm{CT}$ at its origin in order to avoid persistent postoperative lymphatic leak, which is difficult to resolve. The hepatic artery is our preferred site for the end-to-side anastomosis of the branch graft, because of the high risk of complications when the CT is anastomosed in end-to-end fashion.

Then, extensive mobilization of the left renal vein enables control of the right and left renal arteries, as well as the superior mesenteric artery (SMA), which is well visualized in the space over the left renal vein in the mesocolic flexure. An alternative site of SMA preparation is in the omental bursa, as it emerges from behind the pancreas. However, attempting isolation of the SMA at this level may result in pancreatic trauma, which may include injury to the pre-aortic lymphatics with consequent lymphatic leak. After completion of isolation of the renal and visceral vessels, 


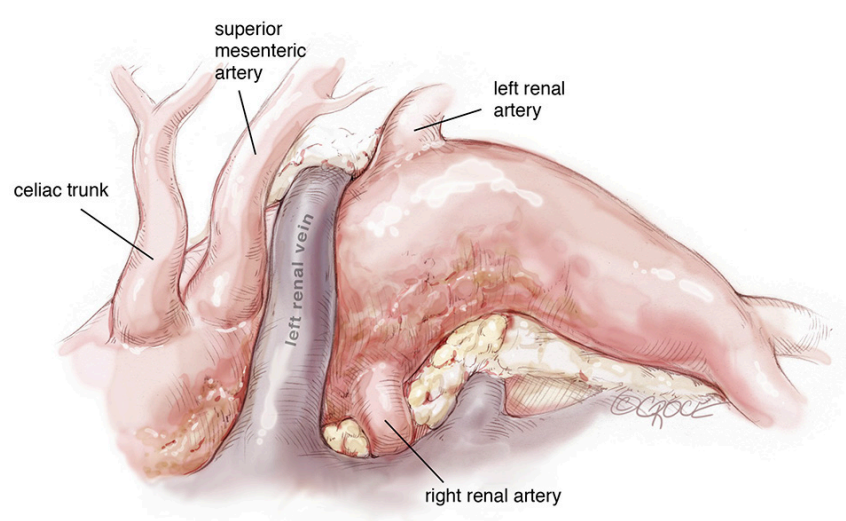

Figure 9 In the second surgical stage a mid-line laparotomy is routinely performed to expose the abdominal aorta and its main branches (the visceral and renal arteries and both common iliac arteries). The extent of the distal aortic replacement (either above or below the iliac bifurcation) depends on the involvement of the common iliac arteries in the aneurysm.

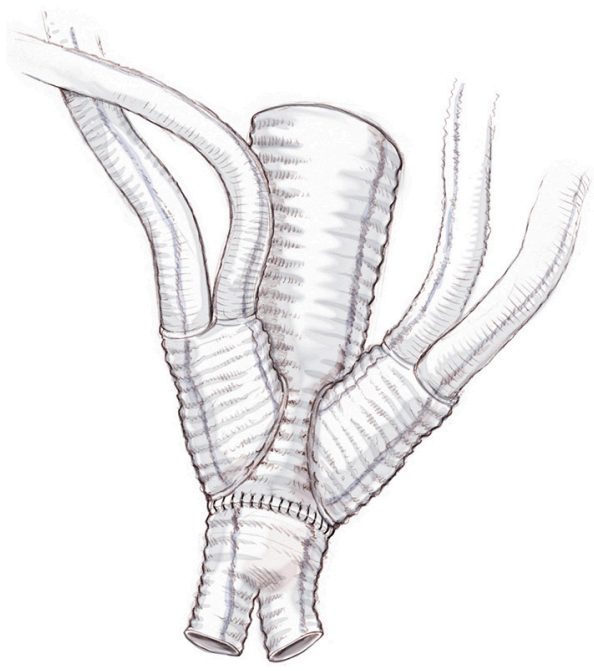

Figure 10 A careful analysis of the imaging and meticulous preoperative planning are critical to choose which strategy will suite better each patient. A dedicate multibranched Dacron graft is then appropriately trimmed.

systemic heparinization is administered and the infrarenal abdominal aorta is cross-clamped together with both common iliac arteries.

The abdominal aorta is opened, back bleeding from the lumbar arteries is controlled and the proximal anastomosis with a multi-branched Dacron graft (i.e., Lupiae graft, Coselli graft or custom-made graft) is performed taking note of the optimal position of the side branches of the prosthesis. The distal aortic anastomosis is often performed on both common iliac arteries, which are involved in the aneurysmal pathology. In light of this, the surgical approach must be determined preoperatively with particular attention paid to the CT angiogram. This enables appropriate sizing of the diameter of the main Dacron prosthesis to be implanted and helps to determine the need to suture a bisiliac Dacron prosthesis to the distal part of the "especially designed" multi-branched Dacron graft (Figure 10). The graft design and its correct orientation are of critical importance in providing an accurate origin and path for the re-routed vessels as well as an adequate length of the Dacron distal landing zone (DLZ) to be used in the subsequent endovascular stage.

After meticulous haemostasis of both main aortic anastomoses, re-routing of the arteries commences with the right renal artery followed by the left renal artery. In the last five years, in order to minimize the incidence of acute kidney injury due to prolonged ischemic time with or without kinking of one or both re-routed renal arteries, the suggested surgical approach has been modified as follows:

* Ligation and partial detachment of the renal artery from its aortic origin;

* Rapid insertion and deployment in its open lumen of an appropriately sized Gore Hybrid Vascular Graft (W. L. Gore and Ass., Flagstaff, AZ, USA) as previously determined by CT imaging;

* Completion of the detachment of the renal arteries from the aorta and suturing with 6-0 polypropylene of its superior and inferior rims to the inserted Gore Hybrid Graft to avoid slipping;

* Renal protection by infusion of $500 \mathrm{~mL}$ of Custodiol HTK solution (Essential Pharma, Newton, PA, USA);

* Completion of renal artery reimplantation by end-toend anastomosis with a 6-0 Gore-Tex suture of the Gore Hybrid Graft to the chosen branch of the multibranched Dacron graft (Figure 11).

SMA re-routing is performed after its ligation in the mesocolon flexure over the left renal vein. Despite its distal connection the right colic artery, SMA ligation in this area enables exclusion of the middle colic artery, which usually continues to supply blood to the transverse colon. Detachment of the SMA in this area is preferred because it avoids pancreatic manipulation and trauma to pre-aortic lymphatics as well as facilitates a Lazy-C orientation of the re-routed vessel. SMA reimplantation should be performed in end-to-end fashion with the interposition of a 7-8 $\mathrm{mm}$ externally reinforced 


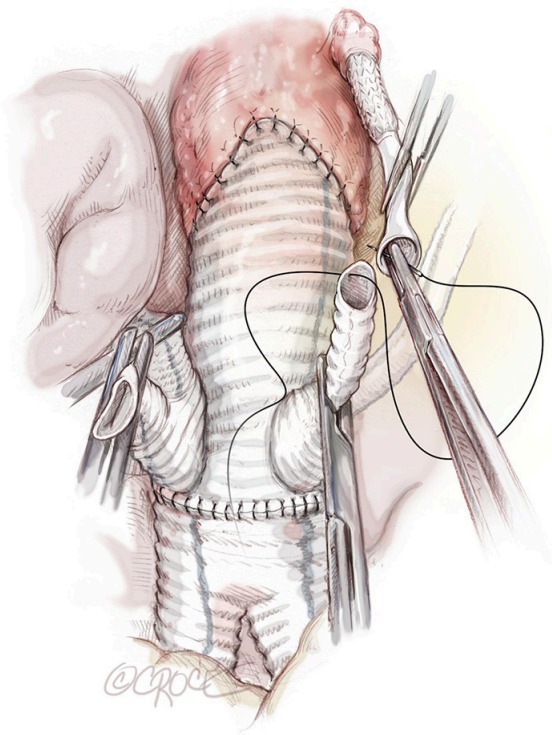

Figure 11 Both the renal arteries are reimplanted performing an end-to-end anastomosis with 6/0 Gore-Tex suture between the Gore Hybrid Graft and the chosen branch of the multibranched Dacron graft.

PTFE vascular graft pre-sewn to the chosen branch of the multi-branched Dacron prosthesis. This seems to avoid lifethreatening acute bowel ischaemia secondary to kinking or occlusion of the re-routed SMA, as we observed in one patient who died after extensive bowel resection.

Celiac trunk re-routing is accomplished after ligation of its aortic origin in the retroepiploic cavity, by an endto-side anastomosis between a pre-sewn $8 \mathrm{~mm}$ externally reinforced PTFE vascular graft and the hepatic artery, thus preserving hepatic, gastric and splenic perfusion through the shaft of the CT. In order to obtain an orientation of the re-routed CT less prone to kinking, we strongly suggest use of an ante-pancreatic Lazy-C approach to perform a safe connection to the common hepatic artery (Figure 12). In our experience, as confirmed from Chiesa et al. (10), the retro-pancreatic passage of the Dacron graft for the CT is an independent risk factor for visceral complications due to the formation of a retropancreatic tunnel resulting in more traumatic manipulation of the pancreas. In addition, the use of a non-externally supported Dacron graft also increases the risk of kinking or occlusion with the retro-pancreatic approach. We like to complete the procedure with the reimplantation of the inferior mesenteric artery, which is deconnected before the infrarenal aortic replacement, in order to fully support the intestinal blood supply. This

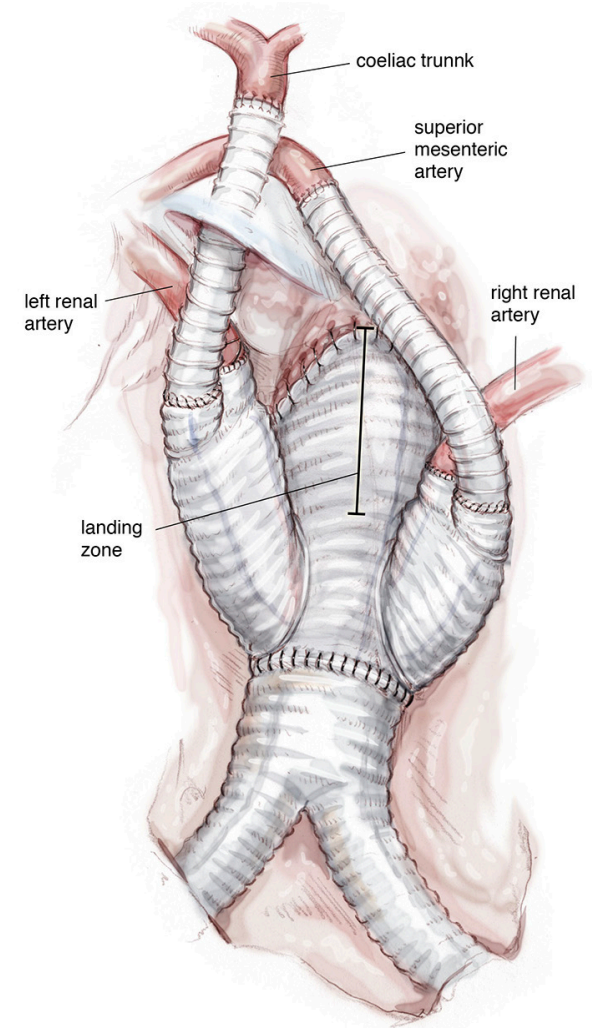

Figure 12 The SMA is generally re-routed toward the Lupiae graft interposing a $7-8 \mathrm{~mm}$ reinforced PTFE vascular graft. The celiac trunk is instead re-routed using an ante-pancreatic Lazy-C approach and performing the distal anastomosis on the common hepatic artery rather than the celiac trunk itself. SMA, superior mesenteric artery.

artery can be anastomosed directly by side-clamping of the main body of the multi-branched prosthesis and this reimplantation should be done whenever feasible.

\section{Completion}

At the end of the renovisceral arterial reimplantation, the rerouted branches are covered with a large pericardial patch to avoid adhesion and compression after bowel repositioning (Figure 13).

\section{Stage III}

\section{Preparation}

The final endovascular repair is carried out 4-8 weeks after the second endovascular stage and is performed in the cathlab with or without general anaesthesia plus trans- 


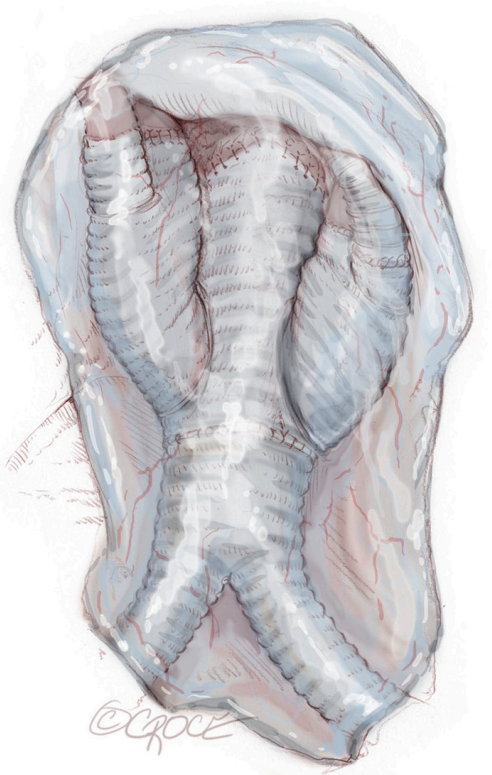

Figure 13 Once all the sutures are checked, the rerouted branches are separated from the bowel by interposing a large pericardial patch, mitigating the risk of adhesion, fistula and compression.

oesophageal echocardiographic monitoring as defined by the cardiac team.

\section{Operation}

The right femoral artery is generally preferred to the left and if possible a totally percutaneous approach is performed over surgical isolation of the femoral artery, depending on the number and the size of the endografts to be implanted, and the relative ratio of iliac to femoral vessels. After heparinization, multiple self-expanding nitinol framed stent grafts are sequentially deployed between the appropriately-sized proximal and the distal DLZ. The ideal site for stent graft release is indicated by the radiopaque markers present on both multi-branched Dacron grafts previously implanted in the ascending and abdominal aorta. The stent grafts are usually oversized 5-10\% more than the DLZ diameters (Figure 14).

\section{Comments}

The hybrid three stage Lupiae technique is an alternative treatment to open surgical repair of MAS type III.

\section{Clinical results}

From January 2006 and December 2016, 27 MAS III

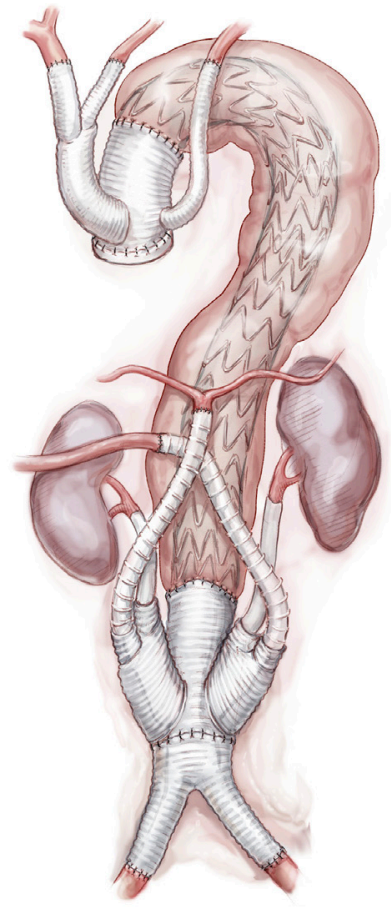

Figure 14 After the final endovascular stage, the residual diseased thoracoabdominal aorta has been excluded by multiple stent-graft implantation.

patients (out of 218 patients affected by different degrees of MAS treated with Lupiae technique) underwent a planned three-stage repair here described. One patient (3.7\%) died 60 days after urgent first surgical stage performed for a retrograde type A aortic dissection complicating a previously diagnosed thoraco-abdominal aortic aneurysm. The cause of death was ARDS. 26 patients underwent the second surgical stage after $58.3 \pm 16.1$ days.

One patient of this group $(1 / 26=3.8 \%)$ died 10 days after the surgical procedure for acute bowel ischemia due to acute occlusion of the re-routed SMA. Another patient showed asymptomatic re-routed celiac trunk occlusion found at follow-up CT scan; however, this patient proceeded to the third endovascular stage without complications. Two patients $(2 / 26=7.6 \%)$ had transient post-operative renal failure after the second stage and were successfully supported by temporary continuous renal replacement therapy. Their endovascular stages were delayed until the 76 th and 83 th postoperative days respectively in order to maximally restore renal function. One patient developed acute descending aortic rupture and a left haemothorax 25 days after the second stage and 
was emergently treated by successful endovascular stent grafting later to be discharged home without major further complications.

\section{Advantages}

The main advantages of the Lupiae technique in treating MAS III patients are:

* Formation of an optimal DLZ in Criado zone 0 or 1 with a length of more than $3.5 \mathrm{~cm}$, thus avoiding a 'bird-beaking' effect and angulation at the endovascular stage;

* Reimplantation of the LSA during the first stage, preservation of the hypogastric artery in the second stage as well as staged closure of the lumbar arteries (in the second stage) and of the intercostal arteries (in the third endovascular stage) support integrity of the collateral network that grants spinal cord perfusion, preventing spinal neurovascular injury $(0 \%$ of paraplegia or paraparesis in our series);

* Avoidance of kinking or compression of the re-routed epiaortic vessels by means of appropriate origin and correct angulation of the branches from the main body of the Lupiae graft and the Lazy C configuration of CT and SMA re-routing together with the use of an externally supported PTFE vascular graft is of paramount importance in preventing the onset of complications.

\section{Caveat}

The Lupiae technique has the following limitations:

* This hybrid approach needs appropriately designed specific vascular grafts to facilitate both surgical and endovascular stages;

* Reno-visceral re-routing to the infrarenal aorta and common iliac artery replacement is a long, demanding surgical procedure;

* Availability of new sutureless telescopic anastomotic techniques with use of self-expanding endografts is expensive, however can significantly reduce operative duration time and minimize surgeon fatigue.

\section{Acknowledgements}

We would like to thank Beth Croce for her medical illustrations.

\section{Footnote}

Conflicts of Interest: The authors have no conflicts of interest to declare.

\section{References}

1. Cullhed I, Lodin H, Malers E. Mega-aorta in a young man. Am Heart J 1960;60:128-33.

2. Rigberg DA, McGory ML, Zingmond DS, et al. Thirtyday mortality statistics underestimate the risk of repair of thoracoabdominal aortic aneurysms: a statewide experience. J Vasc Surg 2006;43:217-22.

3. Quiñones-Baldrich WJ, Panetta TF, Vescera CL, et al. Repair of type IV thoracoabdominal aneurysm with a combined endovascular and surgical approach. J Vasc Surg 1999;30:555-60.

4. Esposito G, Marullo AG, Pennetta AR, et al. Hybrid treatment of thoracoabdominal aortic aneurysms with the use of a new prosthesis. Ann Thorac Surg 2008;85:1443-5.

5. Esposito G, Cappabianca G, Contegiacomo G, et al. Hybrid repair of thoracic and thoracoabdominal aortic aneurysms (mega aortic syndrome) with lupiae technique. Innovations (Phila) 2011;6:366-72.

6. Troisi N, Bichi S, Patrini D, et al. Hybrid three-stage repair of mega aorta syndrome with the Lupiae technique. J Thorac Cardiovasc Surg 2013;145:S171-7.

7. Esposito G, Pennesi M, Bichi S, et al. Hybrid multistep approach to mega-aortic syndrome: the Lupiae technique. Eur J Cardiothorac Surg 2015;47:126-33.

8. Kotha VK, Herget EJ, Appoo JJ. Complications at the Proximal Landing Zone of Endovascular Stent Grafts Deployed in Surgically Replaced Ascending Aorta. Ann Thorac Surg 2016;102:1490-7.

9. Shah AA, Bhattacharya SD, McCann RL, et al. Pan-aortic hybrid treatment of mega-aorta syndrome. J Vasc Surg 2011;53:1398-401.

10. Chiesa R, Tshomba Y, Logaldo D, et al. Possible graftrelated complications in visceral debranching for hybrid $\mathrm{B}$ dissection repair. Ann Cardiothorac Surg 2014;3:393-9.

Cite this article as: Esposito G, Pignatelli A, Contegiacomo G, Cappabianca G, Beghi C, Conte M, De Palo M, Lafranceschina C, Tiecco F, Bartolomucci F, Miccoli M, Conte M. Hybrid three-stage repair of mega aorta with Lupiae technique: tips and tricks. Ann Cardiothorac Surg 2018;7(3):422-430. doi: 10.21037/acs.2018.05.04 